\title{
Active control of properties of concrete: a (p)review
}

\author{
Geert De Schutter (D) Karel Lesage
}

Received: 20 August 2018/Accepted: 10 September 2018/Published online: 20 September 2018

(C) The Author(s) 2018

\begin{abstract}
Concrete properties to a large extent depend on mix design and processing, currently leaving only limited options to actively modify concrete properties during or after casting. This paper gives a (p)review on a more advanced active control of properties of concrete, based on the application of external signals to trigger an intended response in the material, either in fresh or hardened state. Current practices in concrete industry that could be considered as active control are briefly summarized. More advanced active control mechanisms as studied in other fields, e.g. based on hydrogels and other functional polymers, are reviewed and some principles are listed. A specific focus is further given on potential methods for active rheology control. Based on the concepts developed in other fields, substantial progress could be made in order to achieve active control of fresh and hardened concrete properties. However, several challenges remain, like the stability and functioning of the responsive material in a cementitious environment, the applicability of the control signal in a cementitious material, and the economy, logistics and safety of a control system on a construction site or in precast industry. Finding solutions to these challenges will lead to marvelous opportunities
\end{abstract}

G. De Schutter $(\bowtie) \cdot$ K. Lesage

Magnel Laboratory for Concrete Research, Department of Structural Engineering, Faculty of Engineering and Architecture, Ghent University, Ghent, Belgium e-mail: Geert.DeSchutter@ugent.be in general, and for 3D and even 4D printing more particularly.

Keywords Concrete $\cdot$ Active control $\cdot$ Functional polymers $\cdot$ Rheology $\cdot$ Casting $\cdot 3 \mathrm{D}$ printing

\section{Introduction}

The quality and performance of concrete as a construction material depend on mix design, processing and casting. The type, quality and dosage of constituent materials are key elements in view of obtaining the right concrete for the right job. Scientific and technological knowledge concerning the properties of concrete [1], supplemented by long-term practical experience, enable to properly design concrete for a given application, either by relying on prescriptive rules as given in many concrete standards, either by aiming for absolute performance further validated by tests $[2,3]$. General concrete mix design methodologies are available to design concrete compositions based on the properties of the constituent materials, and aiming for specific applications requiring well-defined physical, chemical and/or mechanical properties.

In addition to the mix design, the material properties will be influenced by the mixing process and the casting operation. Mixing procedure and intensity can 
significantly influence the properties of the fresh material $[4,5]$. Casting operations, possibly including high-pressure and/or long-distance pumping, can further influence the material properties [6]. Obvious influences of processing further include (lack of) vibration and (lack of) curing. When execution of casting operations is properly done according to relevant state-of-the-art prescriptions, the aimed-for concrete properties as considered in the mix design, will normally be achieved.

However, many variations, intentionally or not, play an additional role, sometimes making it a hard challenge to achieve intended concrete properties in fresh and hardened state. As the properties of the constituent materials vary in-between deliveries, maintaining constant concrete quality in spite of the changing constituent properties is not always straightforward. This is illustrated in [7] by studying the effect of variable foaming potential of admixtures on the rheological behaviour of paste. Even with identical constituent materials, small variations in mixing and processing can have noticeable effects. A well-known aspect is the influence of variable moisture content and water addition during mixing. In some cases, e.g. some self-compacting concrete mixtures, small variations in water content can invoke unacceptable variations in workability, making the concrete non-robust $[8,9]$. As illustrated in [10], inappropriate rheological properties of the fresh concrete can cause unwanted phenomena like segregation, lack of pumpability, increased formwork pressure, insufficient quality of the surface finish... Undesired extra water addition, excessive air inclusion, insufficient vibration during casting etc. will all have a negative impact on the final mechanical and durability performance of the concrete structure.

Quality control consequently is an important aspect in concrete industry, involving quality control of the constituent materials, quality control of the mixing process, quality control during execution, and quality control (compliance testing) of the final properties. While the quality control actions can be very active in the sense that a lot of people spend a lot of energy in getting things done in the right way, we typically passively rely on the behaviour of the concrete. Once the concrete is mixed and flowing in the pumping pipes or formworks, we have no active methods to change the behaviour of the concrete, e.g. requiring it to flow faster or become stiffer. It is true that pumping pressures could be actively changed, or vibraton methods could be actively modified, but we nevertheless are stuck with a material with a pre-defined rheological response to the external changes. We have no means to modify the material behaviour while processing is ongoing, or when the material is in final position. In other words, we cannot actively change the constitutive behaviour of the concrete, either in fresh or hardened state. We can only change external parameters (e.g. pumping pressure) to invoke an effect (e.g. increase in discharge rate) obeying the constitutive material behaviour.

In other fields, active control methods to change material properties "on demand" do exist. As will be further explained in this paper, special fluids are available responding to an external electric or magnetic trigger signal, changing the behaviour of the material from more fluid-like to more solid-like (or vice versa) on demand [11]. These rheofluids can e.g. be used in smart structures, enabling active intervention for damping of vibrations. Rheological properties like yield stress and viscosity can be actively controlled in a reversible way by applying an external electric or magnetic field. Additional examples of active control of material properties or material actions in other fields will be mentioned further on in this paper, showing very innovative and challenging concepts that will be inspiring for cementitious materials.

Achieving active rheology control (ARC) and active stiffening control (ASC) is the final goal of the ERC (European Research Council) Advanced Grant Project "Smart casting of concrete structures by active control of rheology" (SmartCast) [12]. Active control could also be considered for hardened concrete properties, as will be further illustrated. The concept of active control involves an external action or signal to which the material responds by changing its behaviour. The signal can be given "post-processing", which means after mixing, during the fresh or hardened state. The concept of active control includes the incorporation of special components or advanced (nano- or micro-) materials into the cementitious material that show a desired response to a given specific signal. In literature, the word 'control' is often used, as e.g. in [13] concerning the control of the flow of fresh concrete. Mostly the term 'control' in these contexts refers to efficient changes in mixture composition by adding (pre-processing) new or modified components, like e.g. new generation admixtures, in 
view of obtaining (controlling) desired material properties. Once the cementitious material is mixed, no further intervention is possible to adjust the properties. With active control, we refer to an external signal that can be applied at any time during or after processing of the material, triggering a desired response in the material. The active control can be reversible, or in some cases irreversible.

In this paper, an overview is first given of current practices with active intervention in concrete industry, some of them very traditional, some of them already somewhat pushing the boundaries of current knowledge and understanding. Afterwards, active control mechanisms in other fields are briefly summarized, showing materials, signals and mechanisms. Further focus is then given to methods and applications related to the control of rheological behaviour. Finally, some specific challenges are discussed when transferring existing technologies from other fields to concrete industry in view of enabling active rheology and stiffening control of cementitious materials.

\section{Current practices with active intervention to control concrete properties}

In current concrete practice, active interventions are manifold, although mostly not in the sense of actively modifying material behaviour by triggering a response of mixed-in special components. Nevertheless, an overview of current active intervention methods (as also schematically summarized in Table 1) is a good starting point to learn from, and to push the limit of our current abilities to control fresh and hardened concrete properties.

\subsection{Mechanical intervention}

The direct contact between (aggregate) particles leads to high friction, obstructing the flow. In fresh state, an obvious active mechanical intervention is given by the vibration process, using poker vibration or other techniques. By means of a poker, a vibration with a certain frequency and amplitude is (in this case internally) imposed to the concrete. This "signal" has a certain range of action, triggering the (solid) particles in the cementitious material to respond (vibrate). As a consequence, the yield stress drops to zero within the affected zone [14], and the material liquefies and fills the formwork in a better way (i.e. compacts). While a poker requires an invasive action ('internal vibration'), other vibration methods are available operating from the surface of the concrete, e.g. formwork vibrators. External vibrators on pumping pipes are sometimes used at regular distances in case of pumping of concrete for tall towers, several hundreds of meters upwards.

Vibration of fresh concrete can thus be seen as the active application of an external signal (mechanical vibration with a certain frequency and amplitude), triggering a response from some constituents in the bulk of the concrete (in this case vibration of solid particles), changing the rheological properties of the fresh concrete (reducing the yield stress to zero). In this way, it could be said that active rheology control of fresh concrete is already performed in concrete industry since the 1930s due to the introduction of poker vibrators. However, active rheology control can go much further, as will be illustrated further on in this paper.

\subsection{Chemical intervention}

An active chemical intervention is often applied in case of sprayed concrete, introducing accelerating admixtures in the spraying head. The added accelerating admixture triggers the cement particles, speeding up the hydration process in a chemical way, leading to faster setting (stiffening) and hardening of the sprayed concrete. Similar but even more complex chemical interventions can be applied when at the end of a working day the concrete within a long pumping pipe (e.g. for the construction of a tall tower) is removed for pipe cleaning. The concrete flowing back in a truck mixer can be retarded for a long night by adding a chemical retarder. The next morning, while resuming pumping operations, a chemical accelerator can be added, counteracting the effect of the retarder. This kind of active combination of retarders and accelerators has e.g. been studied by Lesage [15].

The active intervention in this case is giving a chemical signal to the cement particles, leading to a control of the hydration rate. After addition of the accelerator the triggered response of the cement particles is irreversible. However, the triggered response of the added retarder can be reversed by later addition of an accelerator. This kind of active intervention is limited to certain moments in the 
Table 1 Overview of current practices with active intervention to control concrete properties

\begin{tabular}{|c|c|c|c|}
\hline $\begin{array}{l}\text { Type of } \\
\text { intervention }\end{array}$ & "Signal" & "Responsive" constituents & Effect \\
\hline Mechanical & $\begin{array}{l}\text { Vibration (frequency, } \\
\text { amplitude, range) }\end{array}$ & Solid particles (vibration) & Elimination of yield stress (rheology control) \\
\hline Chemical & $\begin{array}{l}\text { Chemical trigger, e.g. } \\
\text { retarder, accelerator, } \\
\mathrm{CO}_{2}\end{array}$ & $\begin{array}{l}\text { Cement particles, molecules in pore } \\
\text { solution } \ldots\end{array}$ & $\begin{array}{l}\text { Retardation or acceleration of hydration reaction } \\
\quad . . \text { (setting and hardening control) }\end{array}$ \\
\hline Thermal & Temperature field & $\begin{array}{l}\text { All constituents (through their thermal } \\
\text { properties), cement particles } \\
\text { (activation energy) }\end{array}$ & $\begin{array}{l}\text { Control of temperature gradients, control of } \\
\text { reaction rate }\end{array}$ \\
\hline Hygral & Moisture & $\begin{array}{l}\text { Pore system (capillary pressure, } \\
\text { diffusion ...), Cement particles } \\
\text { (reactivity) }\end{array}$ & $\begin{array}{l}\text { Control of shrinkage (cracking), control of } \\
\text { hydration process }\end{array}$ \\
\hline Pressure & $\begin{array}{l}\text { Reduced or increased } \\
\text { pressure, or } \\
\text { ultrasound }\end{array}$ & Air & $\begin{array}{l}\text { Control of compaction, contribution to accelerated } \\
\text { curing, dispersion }\end{array}$ \\
\hline Magnetic & Magnetic field & $\begin{array}{l}\text { Magnetizable materials (MNPs, steel } \\
\text { strips or fibres...), water }\end{array}$ & $\begin{array}{l}\text { Set-on-demand, micro-vibration, effect of } \\
\text { magnetized water on hydration, healing on } \\
\text { demand ... }\end{array}$ \\
\hline Electric & $\begin{array}{l}\text { Electric current or } \\
\text { potential }\end{array}$ & Dipolar molecules, ions & $\begin{array}{l}\text { Control of reaction rate (via heating due to } \\
\text { resistivity), formwork release, improved } \\
\text { pumping (via polarization) }\end{array}$ \\
\hline Microwave & $\begin{array}{l}\text { High frequency } \\
\text { electric signal }\end{array}$ & Dipolar elements & $\begin{array}{l}\text { Control of reaction rate (via heating due to internal } \\
\text { dipolar vibration) }\end{array}$ \\
\hline
\end{tabular}

process, e.g. when the concrete is flowing through the spray head in case of sprayed concrete, or when the concrete is in the truck mixer in the case of re-use of concrete in a pumping pipe. The control is no longer possible at other timings, and it is also not possible to adjust the control levels once the admixtures have been added.

Another example of active chemical intervention is the use of carbon dioxide for the accelerated curing of cementitious materials, often in combination with the application of a higher temperature. This active chemical intervention has already been reported by Klemm and Berger [16], but has been given renewed attention in recent years because of environmental issues, as illustrated by Monkman and MacDonald [17], studying the addition of carbon dioxide gas during mixing of the concrete for the production of blocks, and by Nielsen et al. [18], studying carbonatebonded construction materials from alkaline residues.

\subsection{Thermal intervention}

The effect of temperature on the hydration process of cement is well-known, and can be calculated by means of the Arrhenius equation [19], with the activation energy as most important parameter. This knowledge is applied in industry by means of heat curing, e.g. steam cycles in precast industry [20,21]. This involves an active intervention, applying a thermal signal to the hardening concrete in view of accelerating the hydration process and achieving faster setting and strength development (at the cost of reducing somewhat the final strength [22]). The applied thermal field is triggering the cement particles (and/or supplementary cementitious materials) that are responding according to their activation energy, resulting in an acceleration of the hydration process. Steam curing (and heat curing more generally) can be considered as a type of active setting and hardening control.

Another type of active control involving a thermal field is given by cooling measures to mitigate thermal cracking in hardening massive concrete elements [23]. 
In embedded cooling pipes, a cooling agent is circulated through the massive element, imposing lower temperatures inside the hardening concrete $[24,25]$. All constituents of the concrete respond to the applied thermal signal (lower temperature in embedded cooling pipes) following the physical laws of thermal diffusion, as described by Fourier's equation [26]. Important parameters in this process are the specific heat and the thermal diffusivity of the hardening concrete, as well as the heat production due to the exothermal hydration process [27], also responsive to temperature following the previously mentioned Arrhenius Equation.

\subsection{Hygral intervention}

Freshly cast concrete needs to be protected against premature drying, in view of mitigating shrinkage cracking and creating optimal conditions for the hydration process. For onsite casting, protection against premature drying during the first days is for many elements achieved in a passive way by the formworks. After striking of formworks, or for those elements not protected by formworks (e.g. slabs), curing can be performed in an active way by water spraying or other techniques. The active intervention providing sufficient moisture near the concrete surface is interacting with the pore system, based on capillary forces and diffusion processes. As a result, the concrete is maintaining sufficient moisture conditions, mitigating or reducing early age shrinkage cracking, and improving the hydration process [28].

Modern concepts of internal curing by means of porous aggregate particles or super absorbent polymers (SAP) [29] cannot be considered as active interventions, as the water contained in those materials is only released internally as a result of the selfdesiccation linked to the hydration process. In future, however, active intervention could be envisioned, by making SAPs or similar products to release their water content on demand by providing an external trigger. The triggered release of agents contained in hydrogeltype polymers is a known concept in other fields already now, as will be illustrated further on in this paper.

\subsection{Intervention by pressure}

Concrete also includes air, showing a potential effect on the rheological properties depending on the size of air bubbles and the applied shear stresses [30]. During processing, air can influence the rheological behaviour, and vice versa, the processing can influence the air system of the concrete [6]. During mixing, pressures in the mixing pan can be reduced, resulting in vacuum mixing [31]. As a result, the air void system of the fresh (and later on hardened) concrete is influenced, resulting in reduced porosity and increased mechanical and durability performance. Modifying the air content in fresh concrete also influences the rheological behaviour [30]. Others made use of frequently changing pressure waves by means of an inline ultrasound application [32]. A process referred to as cavitational shear disperses cement grains better and thereby reduces the setting time and admixture use. This pre-cast method allows to speed up stiffening and is preferentially applied to neat cement paste, before mixing it as concrete. Vacuum conditions can also be applied during casting, resulting in vacuum compaction of the fresh concrete [33]. While curing, air pressures could be increased, possibly combined with increased temperature and/or increased $\mathrm{CO}_{2-}$ content (see also higher), in view of accelerated hardening of the concrete element [34]. External pressure control can thus trigger changes in air content and pore structure, in view of achieving improved material performance.

\subsection{Magnetic intervention}

Magnetic fields can be relatively easily applied in industry, and can be an element of control during a production process. A magnetic field could be applied to magnetize water before its addition to cementitious materials. A magnetic field changes the physical properties of water, including e.g. specific heat and evaporation rate [35]. Some researchers claim that the use of magnetized water in cementitious materials has an effect on fresh and hardened properties, e.g. Reddy et al. [36]. They observed that the use of magnetized water in concrete resulted in a very significant increase in concrete strength, probably due to the higher surface area of magnetized water. Su and $\mathrm{Wu}$ [37] also observed a strength increase when using magnetized water in mortar and concrete containing fly ash. 
Nevertheless, the use of magnetized water is still open to significant debate, as it is not clear how water could maintain its magnetization long enough after removal of the magnetic field. It has to be further studied whether the reported improvement of properties really is the effect of previous magnetization of water, or rather the effect of other experimental influences that have not been clearly identified.

Soto-Bernal et al. [38] studied the effect of maintained static magnetic fields applied during hardening on the performance of cement paste. They concluded that more $\mathrm{CSH}$ is produced, with denser morphology and reduced porosity. It is conjectured that the magnetic field is influencing the hydration process by means of a restructuration process at molecular scale, resulting in microstructural improvement and increased mechanical performance.

The application of magnetic fields to control rheology of cementitious materials containing magnetic particles in real time has been studied by Nair and Ferron [39]. They considered two types of carbonyl iron powder, added to Portland cement paste (water cement ratio 0.4 ) in dosages up to $10 \%$ by mass of cement (corresponding to $4 \%$ in volume). Magnetic field with strengths up to $1.0 \mathrm{~T}$ have been applied while performing rheological tests on the fresh samples. It is experimentally shown that the rheological behaviour of the paste containing magnetic particles can be actively altered by applying the magnetic field. The effect is depending on the field strength as well as on the dosage of magnetic particles. On the other hand, the authors conclude that the magnetic field does not seem to have a noticeable effect on the morphology and formation of early age hydration product, and does not influence the final compressive strength. This seems to be in contradiction with the previously mentioned conclusions obtained by Soto-Bernal et al. [38], showing that further research is needed to elucidate the effect of magnetic fields on cement hydration and microstructure development. Disregarding the discussion on the effect of magnetic field on hydration, the research of Nair and Ferron [39] is a major step forward towards active rheology control of cementitious materials.

Abavisani et al. [40] also considered magnetic fields to study the possibility to align steel fibres (chips) according to a certain orientation. They conclude that the application of a magnetic field on the fresh steel chip-reinforced concrete facilitates the compaction process, and leads to an increased compressive strength depending on the test direction. Following the optimum direction, the compressive strength increases by more than 17\%. Particularly interesting with regard to rheology control is their observation that the vibration of the steel chips in the alternating magnetic field can be considered as a way to obtain self-compactor elements for concrete.

The same group of authors further studied the effect of alternating magnetic fields and alternating current on the behaviour of reinforced concrete columns [41], considering magnetic fields up to $0.5 \mathrm{~T}$ with frequency of $50 \mathrm{~Hz}$, and $\mathrm{AC}$ current up to $36 \mathrm{~A}$. The alternating magnetic field facilitated the compaction of the fresh concrete in the reinforced columns due to the triggered vibration of the reinforcing bars.

Applying the magnetic field on the hardened concrete seems to lead to contradictory results, with sometimes an increase [40] and sometimes a decrease [41] in performance. The authors state that the application of magneto-electric fields can be the basis for real-time compressive behaviour controlling of reinforced concrete elements, even enabling a control of failure mode. However, their claims are based on contradictory results, and are not fundamentally supported by a clear mechanism explaining the potential influence of the (electro-)magnetic field on the material and structural properties. While the principle of active control of concrete properties by magnetic signals has been set forward, it is not fully clear which mechanism could be the basis for the explanation of the reported effect of magnetization on hydration and microstructure development. This will need further research attention, with more rigorous test protocols in order to fully exclude potential influences of other parameters.

On the other hand, it seems clear that mixed-in magnetic particles or magnetizable materials like steel chips as well as preplaced steel rebars are responding to the magnetic signal, with effects on rheology, compactability and even mechanical performance. This opens a window for new developments and applications, possibly involving new types of magnetizable materials [42] or new fields of application like on-demand healing $[43,44]$. In some applications, the effect of the magnetic field is triggering its response via another physical mechanism like (micro-)vibration (making the link with Sect. 2.1) or temperature increase (making the link with Sect. 2.3). 


\subsection{Electric intervention}

An electric control signal (AC current) has been mentioned in previous paragraph, where it was combined with the research on the magnetic control of the mechanical performance of hardened reinforced concrete columns. Other literature results are available on the application of electric fields on fresh or hardening concrete. As an example, Kovtun et al. [45] apply direct current for the curing of alkaliactivated fly ash concrete. The current serves as an indirect way of heating, while the higher temperature achieved is leading to the accelerated hardening of the material. In their research, Kovtun et al. used a setup in which the direct current, provided through a controllable unit with maximum current $12 \mathrm{~A}$ and voltage up to $260 \mathrm{~V}$, was continuously controlled in order to reach a curing temperature of $60{ }^{\circ} \mathrm{C}$ in the concrete, with an initial heating rate of $20{ }^{\circ} \mathrm{C}$ in order to avoid too fast heating, similar to what is done when applying steam cycles. While the external signal is electric, the physically controlling mechanisms behind the accelerated hardening is the thermal energy. Nevertheless, the response of the alkali activated material to the electric trigger is depending on the activator used, through its influence on the resistivity of the concrete. A similar principle has been followed by Zhang et al. $[46,47]$ as described in their patents concerning the control of setting time of alkali-activated concrete through the application of an electric field. The electric field only serves as a practical means to control the temperature in the cementitious material, depending on its resistivity.

A more innovative application of an electrical field can be found in the papers of Goudjil et al. [48, 49]. They impose an electric potential on a steel formwork panel, up to a level of $3 \mathrm{~V}$ during $10 \mathrm{~min}$, in view of polarizing the concrete layer close to the formwork surface. The applied electric signal triggers a response in the material, resulting in a reduced adhesion between the hardening concrete and the formwork panel. The induced electro-osmotic effect is thus facilitating the formwork release once the concrete has sufficient strength to be self-supporting. In this way, the application of demoulding oils can be avoided.

Inspired by this electrically controlled formwork removal technique, Choi et al. [50] applied a similar polarization technique to improve the flow of concrete in pumping pipes. In a first pilot test, they externally applied an electromagnetic field around steel pumping pipes in order to polarize the material near the pipe surface and improve the formation of the lubrication layer. Although the electromagnetic field applied by Choi et al. indeed seemed to improve the pumping behaviour of the concrete, as experimentally verified by an ultrasound velocity profiling technique, it is important to notice that they did not add any specifically responding material like magnetic particles to the concrete. They merely relied on the response of the classical constituents present in the concrete. Although they mentioned the increased mobility of magnetized water and the results of the pilot test were positive, the fundamental physico-chemical mechanisms behind the success of this technique need further study and elucidation. Understanding this in detail might open the door to the development of new materials that could be mixed-in, strengthening its response triggered by the polarization signal and potentially enabling to better control the response to an intended level.

Another example of electric intervention is the technique of cathodic protection by means of applied current in view of protecting an existing structure from reinforcement corrosion [51]. This technique is becoming more and more popular in concrete practice, and is a nice illustration of currently available active control mechanisms. The fundamental principle of its functioning can be understood by considering a Pourbaix diagram, explaining passive and active states of reinforcing steel in concrete [52].

\subsection{Intervention with microwaves}

As an extension of electric signals, microwaves can also be mentioned in this overview. Microwaves are electromagnetic waves with high frequencies (300 MHz to $300 \mathrm{GHz}$ ) and wavelengths ranging from $1 \mathrm{~mm}$ to $1 \mathrm{~m}$. A dielectric material placed in a high frequency electric field will heat up due to internal friction, as the induced vibration of the dipolar molecules is hindered by molecular attraction forces. As already reported by Xuequan et al. [53], the promoted cement hydration can be attributed to the elevated temperature as a result of the exposure of the hardening concrete to microwaves. However, an additional effect seems to be the desiccation of free water prior to setting, resulting in decreased porosity. As a result, microwave curing of concrete yields very 
high early strength, while also providing slightly higher strength at 7 and 28 days [53, 54].

\section{Active control in other fields}

In other fields, functional materials get a lot of research attention. For a multitude of potential applications, controllable materials are developed, responding to specific trigger signals, and resulting in an aimed-for effect. In this section, an (nonexhaustive) overview is given of some applications, type of materials, type of signals and type of responses. Some specific attention is given to different options to control rheology. The applications and control methods used in other fields can be inspiring in view of reaching active control of properties of concrete.

\subsection{Examples of applications}

A major field of research studying stimuli-responsive materials is given by drug delivery on demand [55-63]. Complex three-dimensional polymer structures (see Sect. 3.2) have been developed that carry drugs or special agents. The polymers are stimuliresponsive, which means that as a result of some trigger signal (see Sect. 3.3) they change their structure or undergo other modifications, resulting in the release of the drug at the intended location in the body. These drug delivery systems have great potential in the fight against cancer and other major diseases. In this context, the development of biosensors can also be mentioned, as they can be integrated within drug delivery systems [64].

Stimuli-responsive polymers are also used for soft robotics and micro-actuators, when the functional polymer can transform chemical energy into a mechanical action like folding or bending [65-69]. Typically, electro-responsive hydrogels are considered for this field of application, as the control is repeatable and reversible. In advanced applications, the stimuli-responsive polymers can be used for the development and control of self-propelled nanomotors [70].

In this non-exhaustive overview of applications of functional materials, reference can also be made to adhesion on demand [71], smart windows [72] and even the possibility to write information into triggered hydrogel by very precise control of the electric signal given to self-assembling molecules [73].

\subsection{Type of polymers}

The majority of current applications relying on stimuli-responsive control is based on hydrogel, generally defined as three-dimensional network structures consisting of polymeric chains joined by tie points or joints and swollen in water up to thermodynamic equilibrium [74]. Hydrogels have already been studied for a long time, with early work in 1930s and 1940s [74]. It is a very broad class of materials that can be synthesized in numerous ways in view of obtaining a wide range of properties and behaviour [75]. The concept of hydrogels is based on crosslinking of hydrophilic polymers into a spatial complex structure that is able to swell or de-swell depending on the thermodynamic equilibrium within the surrounding medium. In case of ionic hydrogels, the ionic interactions bring additional forces that influence the swelling equilibrium. A major parameter is the $\mathrm{pH}-$ value of the surrounding medium, which in current fields of application is typically much lower than in cementitious materials. Nevertheless, hydrogels can be applied in cementitious materials, as illustrated by the well-known application of SAP [29].

Although gels offer great possibilities, they have limitations concerning multi-responsivity and multifunctionality. An alternative approach is the use of vitrimers, covalently crosslinked polymer networks. Vitrimers can be modified in different ways, and even be combined with carbon nanotubes, resulting in many different functionalities, in response to even six different stimuli [76].

A specific group of materials is given by core-shell type particles with monodisperse polymeric cores and uniform shells with independent control of properties of core and shell [77]. Core-shell microspheres dispersed in oils are a nice example of electrorheological fluids that can be used as e.g. dampers, brakes and shock absorbers.

Stimuli-responsive polymers intended for the (reversible) control of shape are also called shape memory polymers (SMP). The mechanism can be based on different controllable transitions, such as reversible molecule cross-linking or anisotropic-isotropic transition. They can be reinforced by different materials, e.g. carbon nanotubes, carbon nanofibers, 
exfoliated nanoclay..., to improve the mechanical performance of the resulting material, called shape memory polymer composites (SMPC) [78]. They could also include $\mathrm{Fe}_{3} \mathrm{O}_{4}$-particles, or other magnetizable materials in order to make them magneto-responsive $[79,80]$.

Magnetic nanoparticles (MNP) can also be incorporated into hyperbranched polymers to make them magneto-responsive. Hyperbranched polymers show good possibilities to make them stimuli-responsive in general, offering good options to include a wide choice of terminal functional groups and branches [81].

The concept and design of stimuli-responsive polymers in general is getting a lot of research attention nowadays. New design strategies [82] and new synthesis and polymerization methods [83] are important aspects in obtaining new stimuli-responsive materials that could also be applicable in cementitious environments, overcoming specific challenges (see Sect. 4) that currently pose problems to most of the existing responsive materials.

\subsection{Type of signals}

Most common signals concern temperature and $\mathrm{pH}$ (or $\mathrm{pH}$ difference), or the application of an electric, magnetic, or electromagnetic field. Many other signals, however, have shown to be applicable in view of inducing a triggered response in functional materials, like e.g. pressure [84], redox-signal [55, 64, 85-88], carbon dioxide [85, 89, 90], light [87, 91], ultrasound [58], UV [92-94] ...

Most stimuli-responsive polymers respond to one or more trigger signals. In case of dual or multiresponsive materials, the desired action depending on the given combination of trigger signals is an important point of attention. In case of two signals, as an example, it could be that the desired response is only activated in the presence of both stimuli, while another option could be the activation in case of any single stimulus. Different other logic combinations can be chosen, as illustrated in [57].

The applicability of the trigger signal depends on the type of material, and further depends on practical considerations. While for some applications photonic signals are convenient and environmentally friendly, the responsive process (often chemical) is typically not showing a high efficiency [95]. For opaque materials like cementitious materials, light signals are not a good option, unless only a response at the surface is needed. Signals based on change in $\mathrm{pH}$ or on electrochemical responses are typically having a higher efficiency. Electric or redox signals further depend on the resistivity or conductivity of the material, and are thus not applicable in all circumstances. Heating and cooling seem easy trigger systems in principle, but are not very practical in many cases.

\subsection{Type of responses}

Roy et al. [96] give an interesting overview of stimuliresponsive materials, with clear illustrations of their effects. Numerous types of responses, resulting in different effects can be listed. In general, control can be reversible or non-reversible, depending on the type of triggered response and the type of signal. A nonexhaustive list of different types of triggered responses is given hereafter:

- Triggering crosslinking resulting in gel formation, often by means of thermally induced sol-gel transition [97]

- Cleavage of polymer molecules, resulting in collapse of a hydrogel or a hyperbranched polymer, or influencing micellization [58, 91, 94]

- Controlling swelling/de-swelling and the release of an agent, due to changes in osmotic pressure by asymmetric distribution of ions in electric field [65], or by change of electronic charge surface potential [61]

- Changing the permeability of a responsive membrane, by closing or opening "chemical gates", e.g. by pH control [96]

- Sorption control of water vapour of e.g. electroresponsive polypyrole film [98]

- Control of solubility, often by changing temperature [99]

- Changing the nature of a complex molecule from hydrophilic to hydrophobic, also enabling control over the micellization process [97]

- Changing the self-assembly, e.g. $\mathrm{CO}_{2}$ and redoxstimulated transformation of entangled worms to rodlike micelles [85]

- Changing the orientation of the polymers, e.g. by the application of an electric and/or magnetic field $[100,101]$ 
- Controlling entanglement of polymer chains [102]

- Controlling aggregation/dispersion of particles in a medium [92]

- Control of trans-cis isomerization of polymers [93]

- Shape-morphing [103]

- Oscillatory shape change, e.g. oscillatory bending [103, 104]

- Crystallization/melting transition [78]

- Crystal anisotropic/isotropic transition [78]

- ...

The listed responses can induce changes in numerous material properties, or can lead to specific actions (like e.g. drug release). Some of the listed responses could have good potential for rheology control. This is further discussed in the following paragraph.

\subsection{Existing methodologies for rheology control}

The rheological behaviour of a material is governed by fundamental physical properties, characterized by specific parameters, further depending on the type of material [105]. For solid materials, the ordering of crystals (in case of crystalline materials) is an important aspect, or the absence of ordering (in case of amorphous or glassy materials). The deformation behaviour, e.g. characterized by elastic properties, is largely depending on the internal mutual interaction forces between crystals and/or amorphous phases. On the other end of the material spectrum, the deformation behaviour of simple liquids (like water) is mainly governed by the atoms or molecules, responding to Van der Waals forces and thermal agitation, and is e.g. characterized by the viscosity which is the only relevant parameter in case of a Newtonian fluid.

When particles are introduced into a liquid matrix, the behaviour can be different depending on the size and nature of the particles, and the resulting interparticle forces. In suspensions, depending on the density of the particles and of the suspending liquid, particles could segregate down- or upwards, as governed by the interplay between gravitational forces and buoyancy forces. However, particles also interact, depending on their concentration in the suspending liquid, counteracting settlement forces. As a result, the viscosity of the suspension will be different from the viscosity of the suspending medium. For low particle concentrations, up to $30 \%$ of the maximum concentration, the viscosity increase is limited. However, at larger concentrations, the viscosity can increase significantly, as described in the Krieger-Dougherty equation or similar models [106]. Furthermore, orientation or alignment of particles can significantly influence the viscosity compared to the case with random orientation of particles.

In case of small particles, in the range of $\mu \mathrm{m}$ and below, colloidal interactions become dominant, mainly Van der Waals forces combined with Brownian motion, and the particles tend to form clusters or aggregates. This aggregation phenomenon could be counteracted by electrostatic repulsion or by steric hindrance, as typically done by superplasticizers in cementitious materials [107]. Depending on the internal structure formation by clustering of particles, the rheological response can be diverse and complex, involving concepts like yield stress (static and dynamic), thixotropy, shear-thickening or shearthinning...

When the particles in the liquid are polymers, droplets or air bubbles, the overall rheological behaviour of the resulting fluid can become even more complex, with additional governing parameters. Although advanced mathematical models are available [108], based on fundamental principles, the experimental characterization of the complex fluids is not an easy task. Measuring rheological properties is not straightforward [109], and rheological constitutive laws are mostly empirical with limited validity. In many research papers, small amplitude oscillatory shear (SAOS) is applied, resulting in the determination of the so-called storage modulus $G^{\prime}$ (representing the energy storage or elastic property of the fluid) and the loss modulus $G^{\prime \prime}$ (representing the viscous dissipation in the fluid) [110]. In case of changing rheological properties of systems with smart functional materials, oscillatory rheometry is an interesting experimental method to study the reversible or non-reversible transition from more liquid-like to more solid-like behaviour.

Considering the governing physics and the resulting characteristic parameters of complex fluids, changing or controlling the rheological behaviour can involve different mechanisms or approaches. A non-exhaustive overview is given hereafter.

A well-known concept to change the rheological properties of a fluid is given by electrorheological (ER) fluids [11, 111, 112] and magnetorheological 
(MR) fluids [113, 114]. Electrorheological response was first reported by Winslow already in 1949 [115]. In these suspensions, electro- or magneto-responsive particles align along the field lines of the applied electric or magnetic field, forming chain- or columnlike structures, changing the rheological properties of the material from liquid-like to solid-like. The transition is reversible, and provides a means to actively control the rheological response of the material, with potential application in many fields of engineering.

In terms of rheological properties, it is reported that the yield stress $\left(\tau_{y}\right)$ in ER fluids is depending on the electric field strength $\left(E_{0}\right)$ in the following way:

$\tau_{0} \propto E_{0}^{a}$

with parameter $a$ typically in the range of 1.5-2.0 [11]. Instead of applying Bingham-type models, a modified constitutive equation, called the Cho-Choi-Jhon (CCJ) model, is often referred to in case of ER or MR fluids [11, 111, 114]. Due to the formation of an internal structure under electric or magnetic field, the loss modulus $G^{\prime \prime}$ typically shows an initial plateau as a function of shear stress, while the storage modulus $G^{\prime}$ is higher than the loss modulus $G^{\prime \prime}$. In electro- or magneto-activated state, the suspension is dominantly showing an elastic behaviour. However, when the shear stress exceeds a critical value, the storage modulus $G^{\prime}$ shows a sudden decrease, physically governed by the rupture of the internal structure and the resulting onset of material flow [11, 111, 114]. SAOS can elucidate in this way the internal structure formation in electric or magnetic fields, providing data in view of accurate electro- or magneto-rheological control.

Besides internal structure formation triggered by electric or magnetic field, other mechanisms can be considered for rheology control. One example is based on micro-vibration or micro-actuation. In Sect. 2.6, it was already mentioned that alternating magnetic field applied to cementitious materials containing steel chips can make the chips work as micro-vibrators [40]. The same principle could be applied to alternating field acting on electro-responsive polymers or polymer composites, that could take the role of microvibrators [66-68].

Coming back to some fundamental parameters governing the rheological behaviour of suspensions and colloids, different triggered responses listed in Sect. 3.4. could also be useful in view of controlling rheological behaviour. Hydrogels can be helpful in sol/gel transitions, release of agents potentially modifying viscosity of the suspending medium, swelling/ de-swelling mechanisms, controlling micellization, shape morphing... The wide range of potential control mechanisms of hydrogels is offering also many opportunities to control rheology of the complex material containing the hydrogels. It is to be mentioned however, that rheology control by means of hydrogels has not been the main point of attention of hydrogel research up to now, while the focus was mainly on other intended applications, like e.g. drug delivery. Any controlled response influencing interparticle forces, particle clustering, viscosity of the suspending liquid, chemistry of the suspending liquid etc. offers a potential mechanism for rheology control. Some examples where rheology control is at least partly reported can be found in literature, like e.g. capillary oil suspensions containing calcium carbonate particles [116], aggregation in aqueous solutions by working on hydrophobicity and micellization [92], controlled solubility by temperature and photocleavage [94], controlled release of nanoparticles [56].

\section{Challenges for active control in concrete}

From a rheological point of view, concrete is a very complex material. It combines features of a colloid and a suspension, while showing at the same time properties of a granular material. In current approaches, reaching desired workability levels in fresh concrete is typically achieved by appropriate mix design, including the use of superplasticizers working on the principle of electrostatic repulsion or steric hindrance [107]. This, however, is a passive way of rheology control, leaving no possibility to adjust workability levels while the concrete is already flowing in a pumping pipe or in a formwork.

Active control is available in some way, as mentioned in paragraph 2. Considering the numerous control mechanisms studied in other fields, as summarized in general and related to rheology more specifically (paragraph 3.), it is clear that new ways could be found to actively control concrete properties in general, and rheological properties of the fresh concrete more specifically. However, many difficulties have to be overcome when applying control mechanisms from other fields to the field of 
cementitious materials. The authors selected three concrete characteristics that are forming the main obstacles for now:

- High pH

- Low signal conductivity

- Low signal transmission

\subsection{High $\mathrm{pH}$}

The fascinating mechanism displayed by different types of hydrogels, as discussed in Sect. 3.2, is tempting to pursue for fresh concrete. However, the existing stimuli responsive hydrogels have been switching between swelling and de-swelling at $\mathrm{pH}$ 's that generally occur in the human body, i.e. $\mathrm{pH} \leq 7.4$. The precarious balance between the fixed charges in the polymer network and the osmotic pressure due to inner mobile ions is sensitive to ion concentration changes in the surrounding fluid. In contrast, a subtle ion concentration change in concrete with a $\mathrm{pH}$ of \pm 12.5 will not be sensed by available weak polybases (pH 7-11) due to the relative abundance of hydroxyl and other ions. Some applications of hydrogels do exist in concrete industry, like the use of SAP, however, without external control features. They could show some autogenous response, e.g. when SAPs are implemented as a method for self-healing [117]. However, further developments are needed to make them respond to active trigger signals to show on-demand responses.

\subsection{Low signal conductivity}

The application of control signals in cementitious materials also comes with some challenges. Some signals will not be applicable, like light signals, as they will not be able to travel easily through an opaque cementitious material. Electric control of hydrogels or other functional polymers is often based on an isolating dielectric medium suspending the functional polymers [112]. In contrast, fresh cementitious materials contain water with a relatively high ionic conductivity and cannot be considered electrically isolating.

\subsection{Low signal transmission}

Another challenge is posed by the large bulk volume and its inert constituents. As an example, while casting a massive foundation slab or a high wall, will it be possible for the trigger signals to reach the bulk of the concrete? With the intended amplitude and/or frequency? In any location? These questions involve different aspects, including fundamental knowledge on the interaction between the physical fields and large volume of concrete in a real environment, involving e.g. pumping pipes and formwork. Furthermore, there are as well practical questions concerning the required installations to provoke the intended fields. Safety issues will become important when working with electromagnetic or other signals on a real construction site.

In some cases, however, bulk control will not be required. As an example, the pumping behaviour to a very large extent depends on the properties of the lubrication layer near the pipe surface [118]. In that case, an economical question might emerge, related to the cost of adding (probably relatively expensive) functional material to the bulk of the concrete, while only needing them for control purposes in a limited zone of interest.

Summarizing the challenges, the following aspects will require due attention when developing active control features for fresh and hardened concrete:

- Stability and functioning of the responsive material in a cementitious environment, typically showing higher $\mathrm{pH}$ values than in other fields

- Applicability of the control signal in a cementitious material, depending on its physical properties like resistivity, conductivity, opacity...

- Economy, logistics and safety of a control system on a construction site or in precast industry

In spite of the many challenges, a good opportunity might be given by digital fabrication methods, including 3D printing. Within the relatively limited volume of a printing head, it will be more practical and realistic to provide control signals to modify the rheology of the fresh cementitious material when moving from pumping line to printed layer. As the rheological requirements in a pumping pipe and for a printed layer are significantly different, an active control of the material properties in the printing head 
would be a very efficient approach. Active control features embedded in 3D printed concrete could open the door to so-called 4D printing in construction industry, as already envisioned by Momeni et al. [119].

\section{Summary}

This paper gives a (p)review on the active control of properties of concrete, based on the application of external signals to trigger an intended response in the material, either in fresh or hardened state. Current practices in concrete industry that could be considered as active control have been listed. More advanced active control mechanisms as studied in other fields, e.g. based on hydrogels and other functional polymers, are reviewed and briefly summarized. A specific focus is further given on potential methods for active rheology control. Based on the concepts developed in other fields, substantial progress could be made in order to achieve active control of fresh and hardened concrete properties. However, several challenges remain, like the stability and functioning of the responsive material in a cementitious environment, the applicability of the control signal in a cementitious material, and the economy, logistics and safety of a control system on a construction site or in precast industry. Finding solutions to these challenges will lead to marvelous opportunities in general, and for 3D and even $4 \mathrm{D}$ printing more particularly.

Funding This paper is a deliverable of the ERC Advanced Grant project 'SmartCast'. This project has received funding from the European Research Council (ERC) under the European Union's Horizon 2020 research and innovation programme (Grant Agreement No. 693755). The authors gratefully acknowledge the financial support received from ERC.

\section{Compliance with ethical standards}

Conflict of interest The authors declare that they have no conflict of interest.

Open Access This article is distributed under the terms of the Creative Commons Attribution 4.0 International License (http:// creativecommons.org/licenses/by/4.0/), which permits unrestricted use, distribution, and reproduction in any medium, provided you give appropriate credit to the original author(s) and the source, provide a link to the Creative Commons license, and indicate if changes were made.

\section{References}

1. Neville AM (2011) Properties of concrete. Pearson United Kingdom, London. ISBN 9780273755807

2. Von Greve-Dierfeld S, Gehlen C (2016) Performance based durability design, carbonation part 1-benchmarking of European present design rules. Struct Concr 17(3):309-328

3. De Schutter G, De Pauw P, Reybrouck N, Caspeele R (2016) Toward absolute durability performance criteria: preliminary case study of a sea lock. J Sustain Cem Based Mater 5(1-2):23-34

4. Dils J, De Schutter G, Boel V (2012) Influence of mixing procedure and mixer type on fresh and hardened properties of concrete: a review. Mater Struct 45:1673-1683

5. Asghari AA, Feys D, De Schutter G (2018) Mix design factors of self-consolidating cement paste affecting the magnitude of variations in rheological properties induced by changes in addition time of PCE-superplasticizer. Constr Build Mater 159:269-276

6. Feys D, De Schutter G, Khayat K, Verhoeven R (2016) Changes in rheology of self-consolidating concrete induced by pumping. Mater Struct 49:4657-4677

7. Han D, Ferron RD (2017) Effect of mixing speed on rheology of superplasticized Portland cement and limestone powder pastes. ACI Mater J 114(4):559-569

8. Van Der Vurst F, Grünewald S, Feys D, Lesage K, Vandewalle L, Vantomme J, De Schutter G (2017) Effect of mix design on the robustness of fresh self-compacting concrete. Cem Concr Compos 82:190-201

9. Asghari AA, Hernandez AML, Feys D, De Schutter G (2016) Which parameters, other than the water content, influence the robustness of cement paste with SCC consistency? Constr Build Mater 124:95-103

10. Ferraris CF, Billberg P, Ferron R, Feys D, Hu J, Kawashima S, Koehler E, Sonebi M, Tanesi J, Tregger N (2017) Role of rheology in achieving successful concrete performance. Concr Int 39(June):43-51

11. Liu YD, Choi HJ (2012) Electrorheological fluids: smart soft matter and characteristics. Soft Matter 8:11961-11978

12. De Schutter G (2016) Smart casting of concrete structures by active control of rheology (SmartCast). ERC Advanced Grant Project 693755, 2016-2021

13. Burns EG, Rieder KA, Curto JW, Tregger N (2018) The rheology of control flow concrete. Concr Int 40(January):43-49

14. Grampeix G, Roussel N, Dupoirier J (2013) Internal vibration and viscous concrete: application and prediction of the radius of action. In: Roussel N, Bessaies-Bey H (eds) Rheology and processing of construction materials, RILEM Proceedings PRO 90. RILEM Publications, pp 123-130. ISBN 978-2-35158-137-7

15. Lesage K (2014) Interactions between cement and combined concrete admixtures. The influence on cement paste rheology. PhD thesis, joint PhD University of Leuven and Ghent University, Belgium

16. Klemm WA, Berger RL (1972) Accelerated curing of cementitious systems by carbon dioxide. Part I. Portland cement. Cem Concr Res 2:567-576 
17. Monkman S, MacDonald M (2016) Carbon dioxide upcycling into industrially produced concrete blocks. Constr Build Mater 124:127-132

18. Nielsen P, Baciocchi R, Costa G, Quaghebeur M, Snellings $\mathrm{R}$ (2017) Carbonate-bonded construction materials from alkaline residues. RILEM Tech Lett 2:53-58

19. De Schutter G, Taerwe L (1995) General hydration model for Portland cement and blast furnace slag cement. Cem Concr Res 25(3):593-604

20. Nurse RW (1949) Steam curing of concrete. Mag Concr Res 1(2):79-88

21. Saul AGA (1951) Principles underlying the steam curing of concrete at atmospheric pressure. Mag Concr Res 2(6): $127-140$

22. Higginson EC (1961) Effect of steam curing on the important properties of concrete. ACI $\mathbf{J}$ Proc 58(9):281-298

23. De Schutter G, Vuylsteke M (2004) Minimisation of early age thermal cracking in a J-shaped non-reinforced massive concrete quay wall. Eng Struct 26:801-808

24. Hedlund H, Groth P (1998) Air cooling of concrete by means of embedded cooling pipes-part I: laboratory tests of heat transfer coefficients. Mater Struct 31(5):329-334

25. Groth P, Hedlund H (1998) Air cooling of concrete by means of embedded cooling pipes-part II: application in design. Mater Struct 31(6):387-392

26. De Schutter G (2002) Finite element simulation of thermal cracking in massive hardening concrete elements using degree of hydration based material laws. Comput Struct 80(27-30):2035-2042

27. Rastrup E (1954) Heat of hydration in concrete. Mag Concr Res 6(17):79-92

28. ACI Committee 308 (2016) Guide to external curing of concrete. ISBN 978-1-942727-87-3

29. Kovler K, Jensen OM (eds) Internal Curing of Concrete, State-of-the-Art Report of RILEM Technical Committee 196-ICC, RILEM Report 41, 2007, 978-2-35158-009-7

30. Feys D, Roussel N, Verhoeven R, De Schutter G (2009) Influence of air content on the steady state rheological properties of fresh self-compacting concrete, without air entraining agents. In: Shi C, Yu Z, Khayat KH, Yan P (eds) Proceedings of the 2nd International Symposium "Design, Performance and Use of Self-Consolidating Concrete (SCC 2009)", Beijing (China), 5-7 June 2009, RILEM Publications PRO 65, pp 287-295. ISBN 978-2-35158073-8

31. Dils J, Boel V, De Schutter G (2015) Vacuum mixing technology to improve the mechanical properties of ultrahigh performance concrete. Mater Struct 48:3485-3501

32. Peters S (2017) The influence of powder ultrasound on setting and strength development of cement suspensions. $\mathrm{PhD}$ thesis, Bauhaus University Weimar. ISBN 978-3-00055602-9

33. Latham J (1979) Vacuum treatment of concrete. ASCE J Civ Eng 8:15-21

34. Yazıcı H, Yardımcı MY, Aydın S, Karabulut AŞ (2009) Mechanical properties of reactive powder concrete containing mineral admixtures under different curing regimes. Constr Build Mater 23(3):1223-1231

35. Wang Y, Wei H, Li Z (2018) Effect of magnetic field on the physical properties of water. Results Phys 8:262-267
36. Reddy VS, Kumar AK, Sumanth A (2017) Effect of magnetic field treated water on fresh and hardened properties of concrete. J Civ Eng Environ Technol 4(2):134-138

37. Su N, Wu C-F (2003) Effect of magnetic field treated water on mortar and concrete containing fly ash. Cem Concr Compos 25(7):681-688

38. Soto-Bernal JJ, Gonzales-Mota R, Rosales-Candelas I, Ortiz-Lozano JA (2015) Effects of static magnetic fields on the physical, mechanical, and microstructural properties of cement pastes. Adv Mater Sci Eng 2015, article ID 934195, 1-9

39. Nair SD, Ferron RD (2014) Set-on-demand concrete. Cem Concr Res 57:13-27

40. Abavisani I, Rezaifar O, Kheyroddin A (2018) Alternating magnetic field effect on fine-aggregate steel chip-reinforced concrete properties. J Mater Civ Eng 30(6):04018087

41. Rezaifar O, Abavisiani I, Kheyroddin A (2017) Magnetoelectric active control of scaled-down reinforced concrete columns. ACI Struct J 114(5):1351-1362

42. Ponsot IMMM, Pontikes Y, Baldi G, Chinman RK, Detsch R, Boccaccini AR, Bernardo E (2014) Magnetic glass ceramics by sintering of borosilicate glass and inorganic waste. Materials 7:5565-5580

43. Joffroy E, Koulialias D, Yoon S, Partl MN, Studart AR (2016) Iron oxide nanoparticles for magnetically-triggered healing of bituminous materials. Constr Build Mater 112:497-505

44. Joffroy E, Bouville F, Bueno M, Studart AR, Partl MN (2018) Iron-based particles for the magnetically-triggered crack healing of bituminous materials. Constr Build Mater 164:775-782

45. Kovtun M, Ziolkowski M, Shekhovtsova J, Kearsley E (2016) Direct electric curing of alkali-activated fly ash concretes: a tool for wider utilization of fly ashes. J Clean Prod 133:220-227

46. Zhang P, Chen K, Wang Y, Liu Y (2016) Alkali-activated cementing material capable of controlling setting time as well as control method and application thereof, Patent CN105731947 A. Published on 6 July 2016

47. Zhang P, Chen K, Wang Y, Liu Y (2016) Concrete construction technique capable of controlling setting time and special equipment therefor, Patent WO2016165413 A1. Published on 20 Oct 2016

48. Goudjil N, Vanhove Y, Djelal C, Kada H (2012) Electroosmosis applied for formwork removal of concrete. J Adv Concr Technol 10:301-312

49. Goudjil N, Djelal C, Vanhove Y, Kada H, Heloun N (2014) Impact of temperature on the demoulding of concrete elements with a polarization process. Constr Build Mater 54:402-412

50. Choi MS, Kim YS, Kim JH, Kim JS, Kwon SH (2014) Effects of an externally imposed electromagnetic field on the formation of a lubrication layer in concrete pumping. Constr Build Mater 31:18-23

51. Chess PM, Broomfield JP (2003) Cathodic protection of steel in concrete. CRC Press, Boca Raton. ISBN 9780203223031

52. Pourbaix M (1976) Atlas of electrochemical equilibrium in aqueous solutions. Pergamon, London 
53. Xuequan W, Jianbgo D, Mingshu T (1987) Microwave curing technique in concrete manufacture. Cem Concr Res 17:205-210

54. Leung CKY, Pheeraphan T (1995) Microwave curing of Portland cement concrete: experimental results and feasibility for practical applications. Constr Build Mater 9(2):67-73

55. Huo M, Yuan J, Tao L, Wei Y (2014) Redox-responsive polymers for drug delivery: from molecular design to applications. Polym Chem 5:1519-1528

56. Kabiri M, Kamal SH, Pawar SV, Roy PR, Derakhshandeh M, Kumar U, Hatzikiriakos SG, Hossain Z, Yadav VG (2018) A stimulus-responsive, in situ-forming, nanoparticle-laden hydrogel for ocular drug delivery. Drug Deliv Transl Res 8:484-495

57. Kabiri M, Ghasemi A, Zangabad PS, Rahighi R, Basri SMM, Mirshekari M, Amiri M, Pishabad ZS, Aslani A, Bozorgomid M, Ghosh D, Beyzavi A, Vaseghi A, Aref AR, Haghani L, Bahrami S, Hamblin MR (2016) Smart micro/nanoparticles in stimulus-responsive drug/gene delivery systems. Chem Soc Rev 45:1457-1501

58. Paris JL, Cabanas MV, Manzano M, Valet-Regi M (2015) Polymer-grafted mesoporous silica nanoparticles as ultrasound-responsive drug carriers. ACS Nano 9:11023-11033

59. Sharma S, Deepak, Kumar A, Afgan S, Kumar R (2017) Stimuli-responsive polymeric hydrogel-copper nanocomposite material for biomedical application and its alternative application to catalytic field. ChemistrySelect 2:11281-11287

60. Moghanjoughi AA, Khoshnevis D, Zarrabi A (2016) A concise review on smart polymers for controlled drug release. Drug Deliv Transl Res. https://doi.org/10.1007/ s13346-015-0274-7

61. Sankoh S, Vagin MY, Sekretaryova AN, Thavarungkul P, Kanatharana P, Mak WC (2017) Colloid electrochemistry of conducting polymer: towards potential-induced in situ drug release. Electrochim Acta 228:407-412

62. Lin CC, Chen YW, Hsiao CS, Chen SY (2017) Electrically responsive $\mathrm{ZnO}$ nanotubes for controlled release of biomolecules. Ceram Int. https://doi.org/10.1016/j.ceramint. 2017.05.299

63. Bijukumar D, Choonara YE, Kumar P, du Toit LC, Pillay $\mathrm{V}$ (2015) An electro-conductive fluid as a responsive implant for the controlled stimuli-release of diclofenac sodium. Pharm Dev Technol. https://doi.org/10.3109/ 10837450.2015.1073742

64. Zhu LZ, Shangguan YG, Sun YX, Li JA, Zhen QA (2010) Rheological properties of redox-responsive, associative ferrocene-modified branched poly(ethylene imine) and its modulation by beta-cyclodextrin and hydrogen peroxide. Soft Matter 6(21):5541-5546

65. Morales D, Palleau E, Dickey MD, Velev OD (2014) Electro-actuated hydrogel walkers with dual responsive legs. Soft Matter 10(9):1337-1348

66. Xu B, Zhang L, Pei YT, Luo JK, Tao SW, De Hosson JTM, Fu YQ (2012) Electro-responsive polystyrene shape memory polymer nanocomposites. Nanosci Nanotechnol Lett 4:814-820

67. Naji L, Safari M, Moaven S (2016) Fabrication of SGO/ Nafion-based IPMC soft actuators with sea anemone-like
Pt electrodes and enhanced actuation performance. Carbon 100:243-257

68. Vogel F, Göktepe S, Steinmann P, Kuhl E (2014) Modeling and simulation of viscous electro-active polymers. Eur J Mech A/Solids 48:112-128

69. Yang C, Liu Z, Chen C, Shi K, Zhang L, Ju SJ, Wang W, Xie R, Chu LY (2017) Reduced graphene oxide-containing smart hydrogels with excellent electro-responsive and mechanical properties for soft actuators. ACS Appl Mater Interfaces 9:15758-15767

70. Tu Y, Peng F, Sui X, Men Y, White PB, van Hest JCM, Wilson DA (2017) Self-propelled supramolecular nanomotors with temperature-responsive speed regulation. Nat Chem 9:480-486

71. Heinzmann C, Weder C, Montero de Espinosa L (2016) Supramolecular polymer adhesives: advanced materials inspired by nature. Chem Soc Rev 45:342-358

72. Chen F, Ren Y, Guo J, Yan F (2017) Thermo- and electrodual responsive poly(ionic liquid) electrolyte based smart windows. Chem Commun 53:1595-1598

73. Yan K, Xiong Y, Wu S, Bentley WE, Deng H, Du Y, Payne GF, Shi XW (2016) Electro-molecular assembly: electrical writing of information into an erasable polysaccharide medium. ACS Appl Mater Interfaces 8:19780-19786

74. Koetting MC, Peters JT, Steichen SD, Peppas NA (2015) Stimulus-responsive hydrogels: theory, modern advances and applications. Mater Sci Eng R 93:1-49

75. Ullah F, Othman MBH, Javed F, Ahmad Z, Akil HM (2015) Classification, processing and applications of hydrogels: a review. Mater Sci Eng C 57:414-433

76. Chen Q, Yu X, Pei Z, Yang Y, Wei Y, Ji Y (2017) Multistimuli responsive and multi-functional oligoanilinemodified vitromers. Chem Sci 8:724-733

77. An JS, Moon IJ, Kwon SH, Choi HJ (2017) Swellingdiffusion-interfacial polymerized core-shell types polystyrene/poly(3,4-ethylenedioxythiophene) microspheres and their electro-responsive characteristics. Polymer 115:137-145

78. Meng H, Li G (2013) A review of stimuli-responsive shape memory polymer composites. Polymer 54:2199-2221

79. Petcharoen K, Sirivat A (2016) Magneto-electro-responsive material based on magnetite nanoparticles/polyurethane composites. Mater Sci Eng C 61:312-323

80. Wang Y, Ye J, Tian W (2016) Shape memory polymer composites of poly(styrene- $b$-butadiene- $b$-styrene) copolymer/liner low density polyethylene $/ \mathrm{Fe}_{3} \mathrm{O}_{4}$ nanoparticles for remote activation. Appl Sci 6:333

81. Wang D, Jin Y, Yan D (2017) Synthesis and applications of stimuli-responsive hyperbranched polymers. Prog Polym Sci 64:114-153

82. Shigemitsu H, Hamachi I (2017) Design strategies of stimuli-responsive supramolecular hydrogels relying on structural analysis and cell-mimicking approaches. Acc Chem Res 50:740-750

83. Moad G (2017) RAFT polymerization to form stimuliresponsive polymers. Polym Chem 8:177-219

84. Steinschulte AA, Scotti A, Rahimi K, Nevskyi O, Oppermann A, Schneider S, Bochenek S, Schulte MF, Geisel K, Jansen F, Jung A, Mallmann S, Winter R, Richtering W, Wöll D, Schweins R, Warren NJ, Plamper FA (2017) 
Stimulated transitions of directed nonequilibrium selfassemblies. Adv Mater 29:1703495

85. Jiang JZ, Zhang DY, Yin JC, Cui ZG (2017) Responsive, switchable wormlike micelles for $\mathrm{CO}_{2} / \mathrm{N}_{2}$ and redox dual stimuli based on selenium-containing surfactants. Soft Matter 13(37):6458-6464

86. Ramtin A, Seyfoddin A, Coutinho FP, Waterhouse GIN, Rupenthal ID, Svirskis D (2016) Cytotoxicity considerations and electrically tunable release of dexamethasone from polypyrrole for the treatment of back-of-the-eye conditions. Drug Deliv Transl Res 6:793-799

87. Wajs E, Nielsen TT, Larsen KL, Fragoso A (2016) Preparation of stimuli-responsive nano-sized capsules based on cyclodextrin polymers with redox or light switching properties. Nano Res 9(7):2070-2078

88. Sen S, Palmore GTR (2016) Stimuli-responsive macromolecular composites: enhanced stress modulation in polypyrrole with redox-active dopants. Macromolecules 49:8479-8488

89. Darabi A, Jessop PG, Cunningham MF (2016) $\mathrm{CO}_{2}$-responsive polymeric materials: synthesis, self-assembly, and functional applications. Chem Soc Rev 45:4391-4436

90. Oechsle AL, Lewis L, Hamad WY, Hatzikiriakos SG, MacLachlan MJ (2018) $\quad \mathrm{CO}_{2}$-switchable cellulose nanocrystal hydrogels. Chem Mater 30:376-385

91. Manouras T, Vamvakaki M (2017) Field-responsive materials: photo-, electro-, magnetic- and ultrasound-sensitive polymers. Polym Chem 8:74-96

92. Du Z, Ren B, Chang X, Dong R, Tong Z (2017) An endbifunctionalized hydrophobically modified ethoxylated urethane model polymer: multiple stimuli-responsive aggregation and rheology in aqueous solution. Macromolecules 50:1688-1699

93. Fang H, Zhao X, Lin Y, Yang S, Hu J (2018) A natural glycyrrhizic acid-tailored light-responsive gelator. Chem Asian J 13:1192-1198

94. He L, Hu B, Henn DM, Zhao B (2016) Influence of cleavage of photosensitive group on thermally induced micellization and gelation of a doubly responsive diblock copolymer in aqueous solutions: a SANS study. Polymer. https://doi.org/10.1016/j.polymer.2016.10.019

95. Ma X, Tian H (2014) Stimuli-responsive supramolecular polymers in aqueous solution. Acc Chem Res 47:1971-1981

96. Roy D, Cambre JN, Sumerlin BS (2010) Future perspectives and recent advances in stimuli-responsive materials. Prog Polym Sci 35:278-301

97. Brassinne J, Fustin CA, Gohy JF (2017) Control over the assembly and rheology of supramolecular networks via multi-responsive double hydrophilic copolymers. Polym Chem 8:1527-1539

98. Okuzaki H, Funasaka K (2000) Electro-responsive polypyrrole film based on reversible sorption of water vapor. Synth Met 108:127-131

99. Huang Y, Yong P, Chen Y, Gao Y, Xu W, Lv Y, Yang L, Reis RL, Pirraco RP, Chen J (2017) Micellization and gelatinization in aqueous media of $\mathrm{pH}$ - and thermos-responsive amphiphilic ABC PMMA $_{82}-b$-PDMAEMA ${ }_{150^{-}}$ $b$-PNIPAM ${ }_{65}$ ) triblock copolylmer synthesized by consecutive RAFT polymerization. RSC Adv 7:28711-28722

100. van der Asdonk P, Hendrikse HC, Sauli AC, Kraaijkamp SPM, Kouwer PHJ (2016) Spatial and temporal patterning of polymers in electric field responsive LC templates. J Mater Chem 4(35):8263-8269

101. Cairns DR, Shafran MS, Sierros KA, Huebesch WW, Kessman AJ (2010) Stimulus-responsive fluidic dispersions of rod shaped liquid crystal polymer colloids. Mater Lett 64:1133-1136

102. Frechet JM (1994) Functional polymers and dendrimers: reactivity, molecular architecture, and interfacial energy. Science 263(5154):1710-1715

103. Jeon SJ, Hauser AW, Hayward RC (2017) Shape-morphing materials from stimuli-responsive hydrogel hybrids. Acc Chem Res 50:161-169

104. Attaran A, Brummund J, Wallmersperger T (2015) Modeling and simulation of the bending behavior of electrically-stimulated cantilevered hydrogels. Smart Mater Struct 24:035021

105. Coussot $P$ (2012) Rhéophysique: La matière dans tous ses états. EDP Sciences, Les Ulis. ISBN 978-2-7598-0759-8

106. Roussel N (ed) (2011) Understanding the rheology of concrete. Woodhead Publishing, Cambridge. ISBN 9780857095282

107. Aïtcin PC, Flatt RJ (eds) (2016) Science and technology of concrete admixtures. Woodhead Publishing, Cambridge. ISBN 9780081006931

108. Barthès-Biesel D (2012) Microhydrodynamics and complex fluids. CRC Press, Boca Raton. ISBN 978-1-13807240-4

109. Feys D, Cepuritis R, Jacobsen S, Lesage K, Secrieru E, Yahia A (2017) Measuring rheological properties of cement pastes: most common techniques, procedures and challenges. RILEM Tech Lett 2:129-135

110. Vega JF, Santamaria A (1998) Small-amplitude oscillatory shear flow measurements as a tool to detect very low amounts of long chain branching in polyethylenes. Macromolecules 31:3639-3647

111. Gao CY, Meng LY, Piao SH, Choi HJ (2018) Hollow submicron-sized spherical conducting polyaniline particles and their suspension rheology under applied electric fields. Polymer 140:80-88

112. Zheng C, Dong Y, Liu Y, Zhao X, Yin J (2017) Enhanced stimuli-responsive electrorheological property of poly(ionic liquid)s-capsulated polyaniline particles. Polymers 9:385

113. Filipcsei G, Csetneki I, Szilagyi A, Zrinyi M (2007) Magnetic field-responsive smart polymer composites. Adv Polym Sci 206:137-189

114. Zhang WL, Choi HJ (2014) Stimuli-responsive polymers and colloids under electric and magnetic fields. Polymers 6:2803-2818

115. Winslow WM (1949) Induced fibration of suspensions. J Appl Phys 20:1137-1140

116. Das AAK, Dunstan TS, Stoyanov SD, Starck P, Paunov VN (2017) Thermally responsive capillary suspensions. ACS Appl Mater Interfaces 9:44152-44160

117. Snoeck D (2015) Self-healing and microstructure of cementitious materials with microfibres and superabsorbent polymers. Doctoral thesis, Ghent University, Belgium. ISBN 9789085788454

118. De Schutter G, Feys D (2016) Pumping of fresh concrete: insights and challenges. RILEM Tech Lett 1:76-81

119. Momemi F, Hassani SMM, Liu X, Ni J (2017) A review of 4D printing. Mater Des 122:42-79 